\title{
HOW EXISTING NLP TOOLS OF ARABIC LANGUAGE CAN SERVE HADITH PROCESSING
}

\author{
Abdelkarim Abdelkader \\ Computer Science Department, Faculty of Computing at Algunfdh, \\ Umm Al-Qura University, KSA. \\ Moath Najeeb \\ Computer Science Department, Faculty of Computing at Algunfdh, \\ Umm Al-Qura University, KSA. \\ Marwan Alnamari \\ Computer Science Department, Faculty of Computing at Algunfdh, \\ Umm Al-Qura University, KSA. \\ Faculty of Computer Science and Information Technology \\ University of Malaya, 50603 Kuala Lumpur, Malaysia

\section{Hamid Malik} \\ Computer Science Department, Faculty of Computing at Algunfdh, \\ Umm Al-Qura University, KSA.
}

\begin{abstract}
The term Natural Language Processing (NLP) refers to all research and development aimed at modeling and reproducing, with the machines, the human capacity to produce and understand linguistic statements with the goal of communication. NLP, which is the crossroads of linguistics, computer science and artificial intelligence, sees its applications, its programs and many computer techniques, at the service of human language in order to apprehend the meaning of data in natural language. A high-level understanding for the human reasoning has long been sought and considered the extreme goal of the early work. for many natural languages such as English and French, and for all levels of analysis, many applications and tools have been developed. Also for Modern Standard Arabic there are several tools using new technologies like artificial intelligence, machine learning, have appeared for decades. These tools generally concern machine translation, information retrieval, ontologies, part of speech tagging, etc. Unfortunately for classical Arabic, language of Quran and Hadith things are just beginning. The purpose of this paper is to show how to benefit from existing tools for Hadih Science processing especially for evaluation and judgment of Hadith to distinguish between the accepted and rejected Hadith.
\end{abstract}


Key words: Arabic Language, Grammar, Hadith Science, NLP Tools, Ontology, Parsers, POS Taggers

Cite this Article: Abdelkarim Abdelkader, Moath Najeeb, Marwan Alnamari, Hamid Malik, How Existing NLP Tools of Arabic Language can Serve Hadith Processing, International Journal of Computer Engineering and Technology 10(6), 2019, pp. 22-31.

http://iaeme.com/Home/issue/IJCET?Volume $=10 \&$ Issue $=6$

\section{INTRODUCTION}

The field of Natural Language Processing (NLP) is organized around questions of modeling the acts of production and perception (recognition, comprehension) of language statements, with a view to their accomplishment or simulation by machines [1]. It constitutes a meeting point between different sectors of knowledge: linguistics, cognitive sciences, experimental psychology; it mobilizes, adapts and contributes to enrich, in addition to models specific to these disciplines, tools borrowed from multiple domains: signal processing, mathematics and theoretical computer science (logic, formal languages), artificial intelligence (representation of knowledge, machine learning) etc.

Natural Language Processing (NLP has as objective creating computer programs that automatically process natural languages. Natural language refers to the language spoken or written by human, as opposed to artificial, computer, mathematical or logical languages. In fact, the treatment does not directly concern the language. But it deals with linguistic data, texts coded in a particular language. Under this generic name we also group written or oral dialogues, and smaller units, such as paragraphs or sentences.

By processing we mean in a first approximation the transformation of an input object into an output object, when it relates to the language. The processing can be of two types:

It can act on linguistic data (i.e. texts) to correct, condense or to translate them. Often, this transformation includes an intermediate step that aims to extract from texts their representations. It is called natural language analysis.

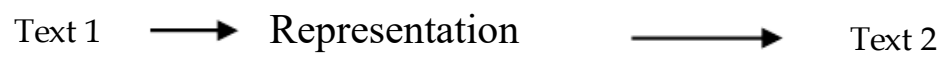

The term representation refers to any translation of the text into a system other than the natural language and makes implicit the explicit information in the text (a set of keywords, a syntactic tree, a logical formula, etc.). In this first type of processing the entry is thus a text and the output a new text or a representation of text.

NLP can also do the opposite operation: it then takes as input the representation of text, to produce a text in natural language. In general, however we do not have the representation of texts but of raw data, like tables, which must first be translated into a representation of text. This operation is called generation of natural languages [2].

\section{Preliminary data}

Representation

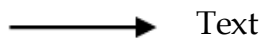

This processing obviously requires various tools that can be grouped into three distinct categories: Linguistic: it describes the various knowledge related to the language. Formal: they express this knowledge in a formalism that is suitable for automatic processing. Finally computer: They use this formal description of knowledge in a concrete computer application so do not be surprised at the diversity of the TAL which involves research in different areas: 
- Computer linguistics and linguistic informatics which develop TAL programs and defines, for this purpose, genuine specialized computer languages for NLP applications.

- Linguistics which provides explicit theories of linguistic knowledge.

- Computer science that optimizes algorithms and treatment programs.

- Mathematics that studies the formal properties of processing tools and theories.

- Artificial intelligence (AI) that deals with the representation of knowledge and their use.

It is classic to present the TALN domain by organizing it in large tasks, with wellidentified input / output: automatic translation, the production of summaries, the generation of statements or texts, the natural language query of databases. data, the synthesis of speech from the text are some examples of these tasks called finalized tasks. In a rough schematization, these final tasks are organized into three main types:

- Tasks related to the production of documents: spell check, text generation from a formal description, question answering system, etc.

- Tasks related to the management of documents: translation, summarization, information retrieval, extraction of information, ontologies, etc.

- Tasks related to the design of human-machine interfaces.

In contrast, intermediate tasks correspond to treatments aimed to the structuring of data in order to reach the degree of analysis necessary for the completion of the final task. The organization of these intermediate tasks takes the prototypical form of the pipeline. This pipeline is organized in large "layers" of processing: segmentation and normalization, lexical, syntactic, semantic, even pragmatic analysis of the statement, during which the initial (linear) representation of the statement is progressively enriched and transformed. From a flow of characters, we thus move on to a flow of words, an analysis tree, a formal representation (logical formula, feature structure, conceptual graph) through enrichment and progressive structuring of the input, each step being a prerequisite to the execution of the next processing step [3].

It soon became apparent that the development of complete language processing systems could not be envisaged without the availability of many sources of knowledge: segmentation rules, lexicons, morphological and syntactic rules, terminological and semantic resources. These sources of knowledge store both linguistic expertise and knowledge representation [4].

This great interest in natural languages, especially Latin language like English, French etc is the cause of the appearance of several NLP about almost all levels of analysis (morphological, syntax, semantic and pragmatic analysis) and dozens of applications (translation, information retrieval, summarization, indexing, information extracting, classification, etc.). In the rest of this article we are going to present the important tools concerning the Arabic Natural Language Processing (ANLP) and how they can serve the Hadith Science [5].

The rest of the paper is organized as follows: we review the different approaches proposed for ANLP in Section 2. We present in Section 3 a linguistic study for Arabic language and Hadith Science. The writing system of the Arabic language is presented. We also present the lexicon and the grammar as well as the inflectional morphology of the classical Arabic and Modern Standard Arabic. Then, we describe the problems of analysis that pose the ANLP .In section 4, we evaluate the existing ANLP tools mainly those related to segmentation, POS 
tagging and syntactic analysis and we will show how to exploit and use them for Hadith Science processing especially for evaluation and judgment of Hadith to distinguish between the accepted and rejected Hadith.

\section{ANLP METHODS}

There are usually two types of methods in this area: stochastic methods and rule-based methods. We can add a third type, the methods based on finite automata or regular expressions, which can be considered as intermediate between robust NLP and theoretical NLP [6].

Rule-based methods, which do not involve counting, are used, for example, to label texts: assign a category, or part of the speech, to each of the words that compose it. If we content to consult a lexicon, there are necessarily ambiguities. For the Arabic language, in a very large proportion, the non voweled words are grammatically ambiguous [7]. For example كَتَّ (kataba, to write) is an accomplished active verb in the third singular person. The shape corresponding non voweled (ktb) admits the five following potential grammatical labels:

- Plural masculine substantive (كُنُبُ kutubun, books).

- Singular masculine substantive (كتْبُ katbun, a writing).

- Accomplished active verb in the third person masculine singular (كَتَبَ kataba, he wrote).

- Accomplished passive verb in the third masculine person singular (كُتِبَ kutiba, he has been written).

- Imperative verb in the second singular masculine person (كَتِبْ kattib, to make write).

Rules that disambiguate the multiplicity of possible categories for a word are called contextual rules because they use the words that precede or follow. In most cases, only the near context is used (neighboring words, at a distance of one, two, or even exceptionally more). These rules can relate directly to words or be more general and relate to categories.

The rule based approach use a knowledge base of rules written by linguists to assign labels to different morphological categories [8] and [9]. We mainly talk about heuristics, contextual rules and non-contextual rules [10]. Decision trees [11] are designed to expose rule bases. A decision tree is a predictive model used to represent classification rules with a tree structure that recursively partitions the learning data set. Each internal node of a decision tree represents a test on a value of a classification attribute, and each branch represents a test result. A prediction is made when a leaf node is reached. This approach is extended to extract and calculate statistical measures used for grammatical labeling.

Stochastic (statistical) approaches form learning models from annotated corpus. They incorporate classification methods such as hidden Markov models to calculate probability rates for each resulting value of a grammatical category of a word. A template can be used to automatically classify other texts by referring to already calculated rates. In a series of words, it is possible to predict the next word, or more precisely to give the probability of appearance of the word that follows. The applications of this hypothesis are multiple, from orthographic correction depending on the context to the recognition of speech, through the assistance to the disabled. The n-grams method consists in giving the probability of appearance of the $n$-th word in function of the preceding (n-1). In practice, the simplification is extreme, since one considers more often than the only word which precedes (diagrams).

A hybrid approach combines linguistic rules with statistical information to resolve morphological ambiguity. In [12], we propose an approach that analyzes grammatical and 
inflectional affixes and grammatical rules based on the MBL (Memory based learning) approach. It is applied to classify a collection of Quranic and educational texts. In [13] combine the rule-based approach with a HMM trigram tagger. The learning of the trigram classifier was done on texts comprising 6000 words. Heuristic rules were applied to select from the proposed results.

Disambiguating linguistic tools are faster and more efficient and reliable than statistical tools [14]. The linguistic approach, which requires only the manual intervention of a linguist, defines a set of rules specific to a particular field. While, the calculated statistics for learning are applied to any test domain. Nevertheless, both statistical and hybrid approaches require learning phase in order to learn the parameters required for disambiguation. Therefore, the hybrid approach is considered to be the most efficient and consistent in terms of analysis because it combines both approaches and benefits from their advantages.

\section{ARABIC LANGUAGE AND HADITH SCIENCE : A LINGUISTIC STUDY}

\subsection{Varity of Arabic Language}

The Arabic language is one of the most spoken and used languages in the world. It is the official language of over 22 countries spoken by more than 320 million people and is used as a vehicle for religious transmission for all 1.5 billion Muslim believers across five continents. It is thus a key element in the culture and thought of a significant part of humanity and world heritage.

In a simplified way, we can say that there are 3 categories of Arabic language, Classical Arabic, Standard modern Arabic and The dialectal Arabic

Classical Arabic : It is a prestigious language associated with religion (Quran and Sunnah). It is used in religious books: books of Hadith, jurisprudence, etc. written texts are usually voweled.

Standard modern Arabic: It is derived from classical Arabic, which has undergone some modifications begun during the last decades of the nineteenth century, the so-called "Nahda period", which means political, cultural and religious revolution. The reformers undertook to modernize the Arabic language by modifying and simplifying the original syntax of the seventh century, but especially by adding modern words. It is associated with literary culture, science and technology, and administrative functions [15].

The dialectal Arabic: It is the language spoken on a daily basis - a language that conveys a whole popular, traditional and contemporary culture. It often has a bad reputation and is perceived as second class. It is a language almost exclusively spoken with varieties of vocabulary between Arabic speakers from different countries.

\subsection{Characteristics of the Arabic Language}

The Arabic language consists of twenty-eight basic letters. Iit is written and reads from right to left. In Arabic script, there is no capitalization and lowercase. Arabic letters change form of presentation according to their position within the words either isolated, initial, median or final. In the Arabic language, all the letters bind themselves except "و", "..", "ذ", "ر", "ز "و and "I" that do not attach to the left. Thus, the Arabic characters having no initial and median form are attached to the character that precedes, but not to the one that follows. An Arabic word consists of consonants and vowels. The vowels are positioned above or below the letters. They are essential for understanding and reading a text correctly [10]. The Arab world uses vowels only for religious or didactic texts (especially for primary schools). Generally, 
newspapers and books do not have vowels. The main encoding of Arabic characters is ISO8859-6 (under Windows it is called: Windows-1256). In addition, Arabic is also supported by Unicode. The Arabic language has a very rich lexicon. It comprises three main categories of words: nouns, verbs and particles, which are subdivided into other sub-categories: preposition, pronoun, conjunction, interjection, article, and adverb. The names and verbs are most often derived from a triliterous root (three consonants). The Arabic language uses about 150 schemes or patterns, some of which are more complex, such as the lengthening of a vowel root (long vowels) or the doubling of a consonant or the addition of one or more elements. A graphic word in Arabic is composed of several complex objects. The basis of this word is called "minimal word". From this word are added other additional components such as particles in the form of extensions. This graphic word becomes "maximal word" when it consists of these elements: proclitic, prefix, base, suffix, enclitic [16]. So, The Arabic language is a strongly agglutinated language in the sense that words can be formed from a base to which we can add affixes and clitics. The construction of sentences in Arabic is flexible. The order of words in a given sentence is relatively free. Generally, a word placed at the beginning of the sentence is a term on which we want to attract attention, follows the longest term or the richest sense or sound. This flexibility causes artificial syntactic ambiguities due to the consideration of all the possible combination rules of the components of a sentence.

\subsection{Hadith and Isnad Overview}

Science of Hadith is a science of referential sources and rules, by which the state of the chain of transmitters of information and the statement of information is known, so as to accept or reject it. Its domain is the chain of transmitters and the statement of traditional information to accept or reject it. Its interest is to discern the authentic of the defaulter among the Ahadith.

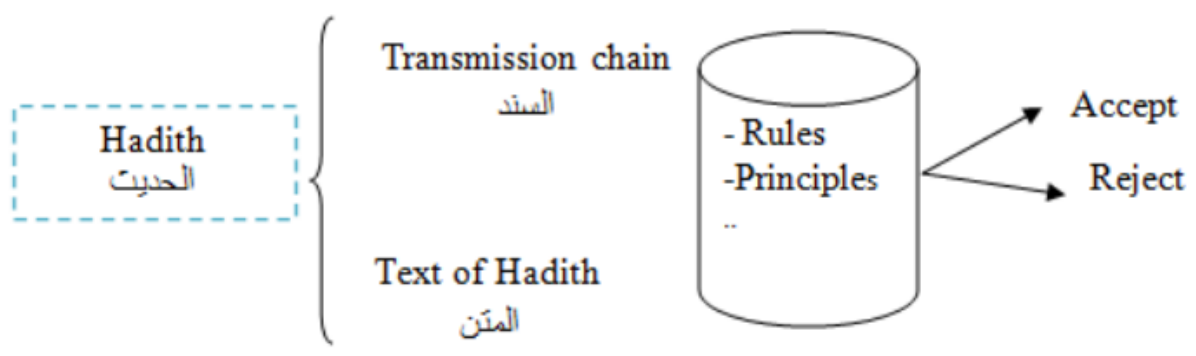

Figure 1 Objective of Hadith Processing

The term Hadith (the plural is Ahadith) is defined as follow : "Everything attributed to Prophet Muhammad (or the Companions or following generation), such as words, deeds, explanations, or characteristics of his creation and character" [17]. Each Hadith is composed of three parts as is shown in Figure 2. 


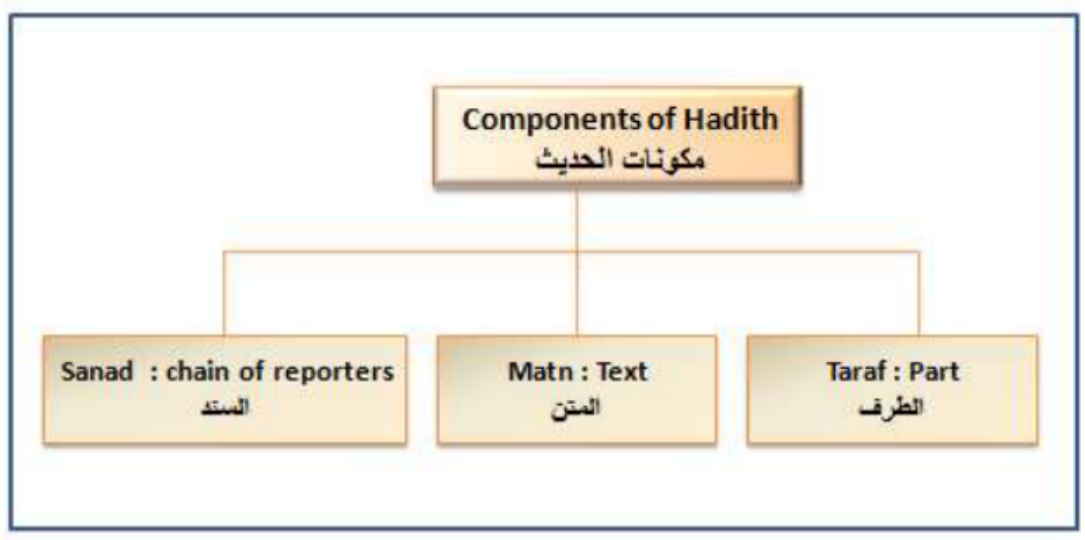

Figure 2 Components of Hadith

The objective of Hadith processing is the classification of Hadith to distinguish between accepted and rejected Hadith. All Knowledge about Hadith science and Isnad (classification of Hadith, structure of Isnad, lexicon, narrator's information, Isnad grammar) are presented in [17], [18] and [19].

\section{ANLP TOOLS AND HADITH PROCESSING}

NLP is a domain of knowledge and methods developed around various concerns. Many concepts and techniques govern its study and it is at the intersection of multiple disciplines: theoretical computing, logic, linguistics, artificial intelligence, but also neuroscience, statistics, etc. The sequence of operations that must be followed to succeed in the complete and automatic comprehension of a sentence is as follows:

1. Segment the sentence into lexical units (words) : At this level will appear the problem of separator, named entities, numbers, etc. for the processing of Hadith isnad this problem is even worse because the segmentation must be done according to the essential components of أحمد بن إبر اهيم ابن خالد الموصلي , حدثنا، أخبرنا، سمعت narrator name like (أبو علي (the not word by word. Then, identify the morphological components, and their properties: This is the morphological analysis step.

The morphological analyzer consists after segmentation of the text, to study the form of an isolated word (without context) and to deduce the derivational and inflectional information. Thus, the analyzer must generate for the word treated one or more morphological solutions described by the following information: the suffixes, the prefixes, the radical, the canonical form (lemma) as well as other information such as the grammatical gender (feminine, masculine), the number (singular, plural) or the time (present, perfect past, ...). For the Arabic language, there are several of analyzers that require linguistic resources (corpus, basic lexicon, text segmenters in sentences, identification of sentence...). For this reason, most of the work has been based on morphological labeling based on machine learning.

Aramorph: Developed by Tim Buckwalter [20] in Perl language for the Linguistic Data Consortium, University of Pennsylvania (LDC) currently in Java. This analyzer is considered "the most respected lexicological resource of its kind". The analyzed input text must be transformed into ASCII (transliteration) code before processing and the analysis result must be transcribed in Arabic (inverse transliteration) in order to be understood. this analyzer can be exploited to process any Sanad.

Sebawi: It is a morphological analyzer of the Arabic language. It is a surface analyzer used in information retrieval applications. He only realizes the search for the possible roots of an Arabic word. This morphological analyzer arrives in $84 \%$ of cases to successfully find the 
root [21]. This analyzer can be used to process Hadith text in order to construct in information system for Hadith science.

APT tagger : This POS tagger is based on a combination of statistical techniques and rulebased techniques [22]. The tag tags of the APT are initially derived from the tagset of the BNC corpus (British National Corpus), but have been modified taking some concepts of traditional Arabic grammar. The reason for this change is that the Arabic language has its own syntactic, semantic and morphological systems that make it difficult to adapt to the "tagset" of Indo-European languages. This is considered the most robust among several other bases either on rule-based approach or stochastic approach and can be exploited for Hadith Isnad.

2. Identify higher-level constituents (group), and the relations (of dominance) that they maintain between themselves: this is the stage of syntactic analysis. For this step, we recommend to use the parser presented in [23] and [24]. Figure 3 shows a part of the CFG of the parser presented in [24].

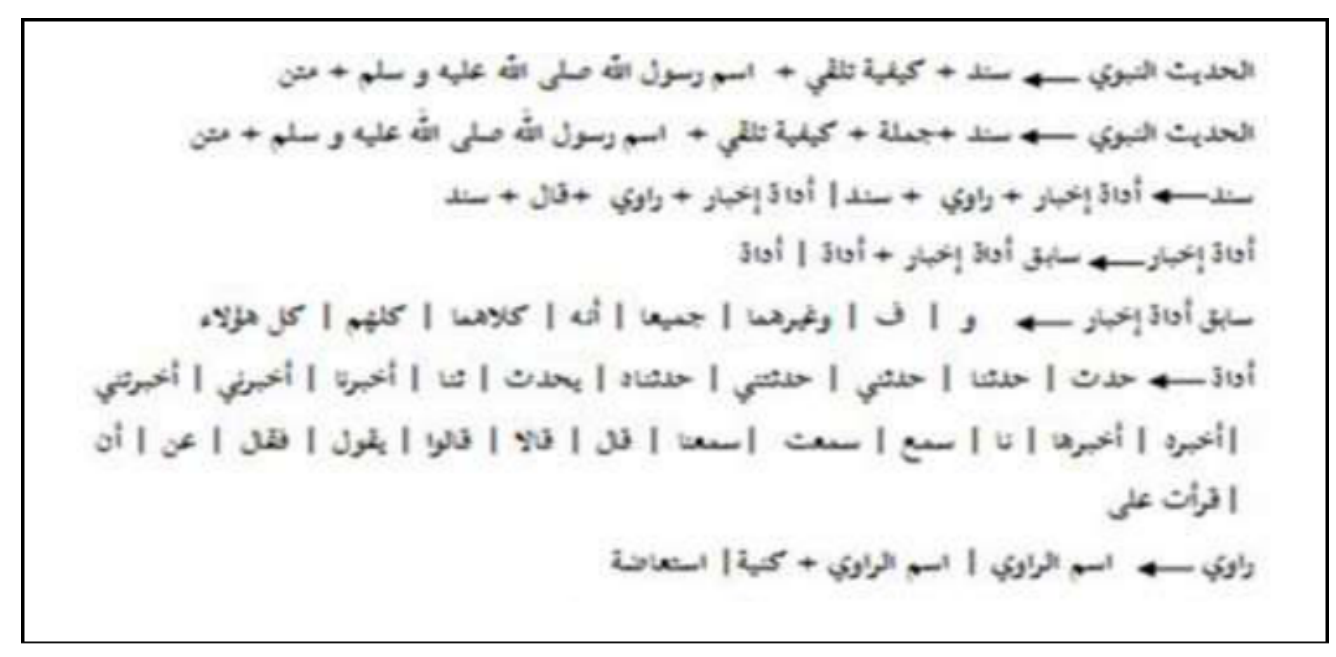

Figure 3 Part of CFG grammar for Hadith Isnad

3. Construct a representation of the meaning of this sentence, by associating with each evoked concept an object or an action in a world of reference (real or imaginary): it is the stage of semantic analysis.

4. Finally recognize the function of the statement in the particular context of the situation in which it was produced: this is the pragmatic stage step.

This two levels (semantic and pragmatic analysis) concern essentially the processing of text of Hadith in order of classification, information retrieval, information extracting and ontology construction [25].

\section{CONCLUSION}

The study of natural language and the mechanisms necessary for its automatic processing by machines is a field of study abounding, and rich in potential or emerging applications. Much progress remains to be made to better understand this faculty and to build systems capable of supporting comparison with humans, but the state of knowledge today allows us to propose many effective solutions to real problems and demands. In this paper, we are interested in presenting a state of the art on the approach, methods and tools for the Arabic language processing. Although several works exist on this task, the most existing tools are not reproducible because they are partially described and the systems are not available. Similarly, it is difficult to evaluate the results and to compare them because the evaluation methods are not clearly presented. One of the limitations of practically all the sophisticated processing 
systems is that they use a large amount of expert knowledge: lexicons, grammar rules, semantic networks ... This partly explains why it does not exist processing system that is both complete (ie integrating all the processing levels) and independent of the domain (ie able to process with any efficiency any type of text). There is another, less visible, reason which limits the progress of progress in NLP, which is the great number of phenomena, the state of linguistic knowledge is insufficiently formalized for use by NLP system designers.

Despite that, we conducted in this paper a thorough study about ANLP methods : rules based methods and stochastic methods and we have specified for each case of processing what is the appropriate method. Then we present a linguistic study for Arabic language and Hadith Science. The writing system of the Arabic language is described. We also presented the lexicon and the grammar as well as the inflectional morphology of the classical Arabic and Modern Standard Arabic. Then we selected a set of ANLP tools that we can exploit in the segmentation, in the part of speech tagging of Hadith isnad or in the development of parser. Other ANLP tools and methods can be used in the processing of text of Hadith in order of information retrieval or extracting, classification, summarization and ontology construction.

\section{REFERENCES}

[1] Dale H. and Harold S. Handbook of Natural Language Processing. Marcel Dekker, 2000.

[2] Daniel J. and James H. Speech and Language Processing. Prentice Hall, 2000.

[3] Manning, C. and Schütze, H. (1999). Foundations of Statistical Natural Language Processing. The MIT Press, Cambridge, Massachusetts.

[4] Zheng X. and Chen H. (2013). Deep learning for chinese word segmentation and pos tagging. In Proceedings of the 2013 Conference on Empirical Methods in Natural Language Processing, p. 647-657 .

[5] Habash N. and Owen R. 2005. Arabic tokenization, part of speech tagging and morphological disambiguation in one fell swoop. In Proceedings of the Association for Computational Linguistics (ACL'05).

[6] Hosny A., Shaalan K. and Fahmi A. (2008). Automatic morphological rule induction for Arabic. In Proceedings of the Workshop on Human Language Translation and Natural Language Processing within the Arabic World (LREC'08). 97-101.

[7] Abdelkarim A., Dalila S. and Rafik B. A categorization algorithm for the Arabic language, International Conference on Communication, Computer and Power (ICCCP'09), Muscat, February 2009.

[8] Daoud D. (2009). Synchronized Morphological and Syntactic Disambiguation for Arabic. Advances in Computational Linguistics 41, 73-86.

[9] Othman E., Shaalan K. and Rafea A. (2004). Towards Resolving Ambiguity in Understanding Arabic Sentence. In the proceedings of International Conference on Arabic Language Resources and Tools, NEMLAR, Egypt, 118-122.

[10] Farghaly A. and Shaalan K. Arabic natural language processing: challenges and solutions. ACM Trans. Asian Lang. Inform. Process. 8, 4, Article 14, 22 pages, December 2009.

[11] Shaalan K. Rule-based approach in Arabic natural language processing, International Journal on Information and Communication Technologies, Vol. 3, No. 3, June 2010.

[12] Tlili-Guiassay Y. (2006). Hybrid Method for Tagging Arabic Text. Journal of Computer Science 2(3): 245-248.

[13] Zribi C. and Ben Ahmed M. (2006). An Efficient Multi-agent System Combining POSTaggers for Arabic Texts. In Proceedings of 7 th international conference of 
Computational Linguistics and Intelligent Text Processing, LNCS Volume 3878, Springer, 121-131.

[14] Hoceiny Y., Cheragui A. and Abbas M. (2011). Towards a New Approach for Disambiguation in NLP by Multiple Criterian Decision-Aid. The Prague Bulletin of Mathematical Linguistics 95, 19-32.

[15] Badawi E., Carter M. and Gully A. 2004. Modern Written Arabic: A Comprehensive Grammar. Routledge, London.

[16] Moath, N., A. Abdelkarim and A. Musab, 2014. Arabic natural language processing laboratory serving Islamic sciences. Int. J. Adv. Comput. Sci. Applic., 5: 114-117. DOI: 10.14569/IJACSA.2014.050316

[17] Moath, N., A. Abdelkarim, A. Musab and O. Abdelrahman, 2015. A lexicon for Hadith science based on a corpus. Int. J. Comput. Sci. Inform. Technol., 6: 1336-1340.

[18] Moath N. 2014. Towards innovative system for hadith isnad processing. Int. J. Comput. Trends Technol., 18: 257-259. DOI: 10.14445/22312803/IJCTT-V18P154.

[19] Moath N. XML Database for Hadith and Narrators, American Journal of Applied Sciences, vol. 13, no. 1, pp. 55-63, 2016.

[20] Buckwalter T. Arabic Morphological Analyzer Version 2.0 LDC2004L02. Web Download. Philadelphia: Linguistic Data Consortium, 2004.

[21] http://www.qamus.org/morphology.htm.

[22] Darwish D. Building a shallow Arabic Morphological Analyser in one day. Proceedings of the ACL-02 workshop on Computational approaches to Semitic languages, Philadelphia, Pennsylvania.

[23] Shereen Khoja. 2001. APT: Arabic Part-of-speech Tagger. Proc. of the Student Workshop at NAACL 2001.

[24] Eman O. Khaled F., and Ahmed R., A Chart Parser for Analyzing Modern Standard Arabic Sentence. MT Summit'IX Workshop: Machine Translation for Semitic Languages, USA, 2003.

[25] Moath N. Multi-agent system for hadith processing. International Journal of Software Engineering and Its Applications 9, 9, pp. 153-166, 2015.

[26] Bounhas I. On the Usage of a Classical Arabic Corpus as a Language Resource: Related Research and Key Challenges. Published in ACM Trans. Asian \& Low, DOI:10.1145/3277591, 2019. 